\title{
Electrical and optical characterization of conducting poly-3-methylthiophene film by THz time-domain spectroscopy
}

\author{
Tae-In Jeon ${ }^{\mathrm{a})}$ and D. Grischkowsky ${ }^{\mathrm{b})}$ \\ School of Electrical and Computer Engineering and Center for Laser and Photonics Research, Oklahoma \\ State University, Stillwater, Oklahoma 74078
}

\author{
A. K. Mukherjee and Reghu Menon \\ Department of Physics, Indian Institute of Science, Bangalore, India
}

(Received 22 August 2001; accepted for publication 11 October 2001)

\begin{abstract}
Using THz time-domain spectroscopy (THz-TDS), we have measured the absorption and dispersion of $7.5 /(\Omega \mathrm{cm})$ conducting poly-3-methylthiophene film from low frequencies up to $4 \mathrm{THz}$. From these measurements the complex conductance that increases with increasing frequency was obtained over this frequency range. The results do not follow simple Drude theory and are not comparable with normal metal and semiconductors; the results were well fit by the localization-modified Drude theory. () 2001 American Institute of Physics. [DOI: 10.1063/1.1427754]
\end{abstract}

The frequency-dependent complex conductivity of conducting polymers is one of the most important properties of these materials, ${ }^{1-4}$ associated with the 2000 Nobel Prize in Chemistry. THz time-domain spectroscopy (THz-TDS) has recently been shown to be the ideal tool to characterize the carrier dynamics in conducting polymers, ${ }^{3}$ similar to the previous THz-TDS characterizations of semiconductors. ${ }^{5-10}$ It is not possible to electrically characterize conducting polymers by simple electrical measurements using mechanical contacts, e.g., Hall effect measurements. For such characterization ohmic contacts would have to be fabricated on the polymer itself. The previous far-infrared Fourier transform spectroscopy reflectivity measurements for conducting polymers require the Kramers-Kronig analysis to calculate the absorption and dispersion of the samples. ${ }^{1,2}$ In our THz-TDS measurements we directly measure both the absorption and dispersion of the conducting polymer film.

In the simple Drude theory picture of conduction the key parameters describing the dynamics of free carriers in a material are the plasma frequency $\omega_{p}$ and the carrier damping rate $\Gamma=1 / \tau$, where $\tau$ is the carrier collision time. Because $\omega_{p}$ and $\Gamma$ characteristically have $\mathrm{THz}$ values, measurements spanning the values of these parameters must be performed in the THz frequency range. ${ }^{3,5-10}$ The experiments described in this letter present such THz-TDS measurements on a free standing $15 \mu \mathrm{m}$ thick film of $7.5 /(\Omega \mathrm{cm})$ low conductivity poly-3-methylthiophene, prepared electrochemically at $-40{ }^{\circ} \mathrm{C}$. Our results determine, for the first time, the absorption and index of refraction from low frequencies to beyond $4 \mathrm{THz}$. The fact that the measured frequency-dependent absorption and index of refraction are mostly due to the free carriers allows the complex conductivity to be determined from the measurements over the full frequency range. In contrast to the previous THz-TDS measurement of the 215/ $(\Omega \mathrm{cm})$ high conductivity polypyrrole which was well fit by Drude theory, ${ }^{3}$ here for a 30 times lower conductivity $7.5 /$

\footnotetext{
a) Permanent address: Korea Maritime University, Division of Electrical and Electronics Engineering, Pusan, Korea.

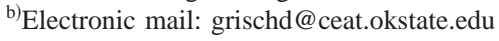

$(\Omega \mathrm{cm})$ sample of poly-3-methylthiophene, the results can only be fit by the localization-modified Drude theory.

For the THz-TDS system, a GaAs transmitting antenna with a simple coplanar transmission line geometry and a silicon on sapphire receiving antenna consisting of a micron size dipole antenna embedded in a coplanar transmission line are optoelectronically driven by $16 \mathrm{~mW}$ average power beams of 65 fs pulses from a mode-locked Ti:sapphire laser with an $86 \mathrm{MHz}$ repetition rate. The THz-TDS characterizations were performed by first measuring the transmitted pulses with no sample in place as shown in Fig. 1(a). The freely propagating $\mathrm{THz}$ electromagnetic pulses were then compared to the measured $\mathrm{THz}$ pulses transmitted through the thin polymer film under investigation as shown in Fig. 1(b). Analysis of the respective numerical Fourier transforms determines the frequency-dependent absorption and index of refraction. Figure 1(c) shows the two $\mathrm{THz}$ pulses in the same time domain with the output $\mathrm{THz}$ pulse multiplied by 4 to be on the same relative $y$-axis scale. From this accurate measure of the relative time delay $\Delta t=135 \mathrm{fs}$ between the two pulses, we obtain a relatively good estimate of the index of refraction $n_{r}=3.65$ of the sample from the simple relationship $\Delta t=\left(n_{r}-1\right) L / c$. The normalized amplitude spectrum of the input reference pulse is shown as the top curve in Fig. 1(d) together with the spectrum of the transmitted output pulse shown as the lower curve on the same relative scale. Although the reference spectrum has useful amplitude up to 5 $\mathrm{THz}$, the output spectrum has only a $4 \mathrm{THz}$ bandwidth. Because of the very thin sample, the multiple reflections are overlapping the main $\mathrm{THz}$ pulse. The multiple reflection effect is shown by the oscillation of the transmission coefficient displayed in the inset of Fig. 1(d). Here the free spectral range (FSR) of our $L=15 \mu \mathrm{m}$ thick film (etalon) with an approximate index of refraction $n_{r} \approx 3.65$ is FSR $=c /\left(2 L n_{r}\right) \approx 2.7 \mathrm{THz}$, which is in good agreement with the peak of the oscillation, especially considering that the index is not constant.

For our experimental situation with a relatively small total absorption and a thin sample, the multiple reflection of the $\mathrm{THz}$ pulse occurs between the two surfaces of the 

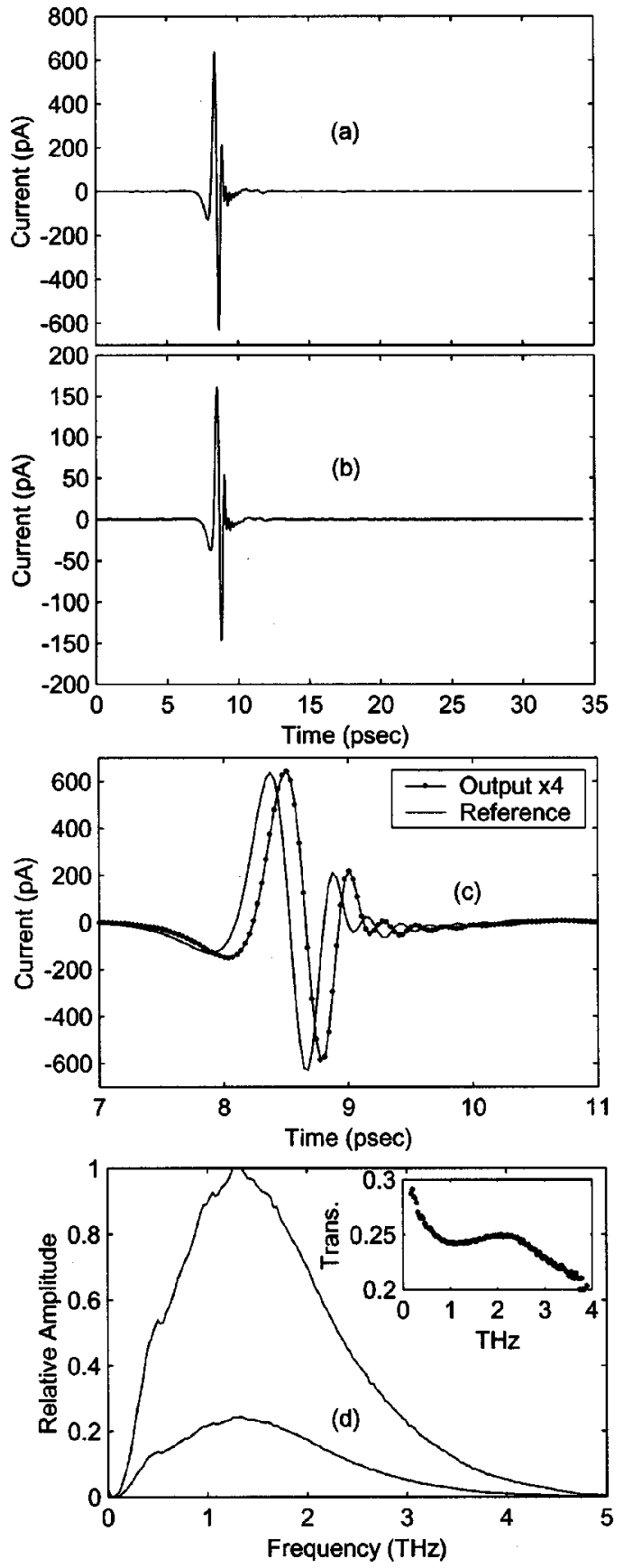

FIG. 1. (a) Measured reference input $\mathrm{THz}$ pulse. (b) Measured output $\mathrm{THz}$ pulse transmitted through sample. (c) Expanded views of the two $\mathrm{THz}$ pulses with the output $\mathrm{THz}$ pulse multiplied by 4 times. (d) Amplitude spectra of input pulse and transmitted pulse; inset, measured amplitude transmission coefficient.

sample. For this situation the frequency-dependent complex amplitude transmission $t(\omega)$ is given by ${ }^{11}$

$$
\begin{aligned}
& t(\omega)=t_{12} t_{21} \exp (-\alpha L / 2) \exp (i \beta) / \\
& {\left[1+r_{12} r_{21} \exp (-\alpha L) \exp (i 2 \beta)\right] .}
\end{aligned}
$$

$t_{12}$ and $r_{12}$ are the complex Fresnel transmission and reflection coefficients from air into the sample of length $L ; t_{21}$ and $r_{21}$ are the transmission and reflection coefficients from the sample into air; the propagation vector is $\beta=2 \pi n_{r} L / \lambda_{0} ; \alpha$ is the power absorption coefficient $\alpha=n_{i} 4 \pi / \lambda_{0}$ with $n_{i}$ designating the imaginary part of the index. By THz-TDS we can measure the magnitude and phase difference of the com-

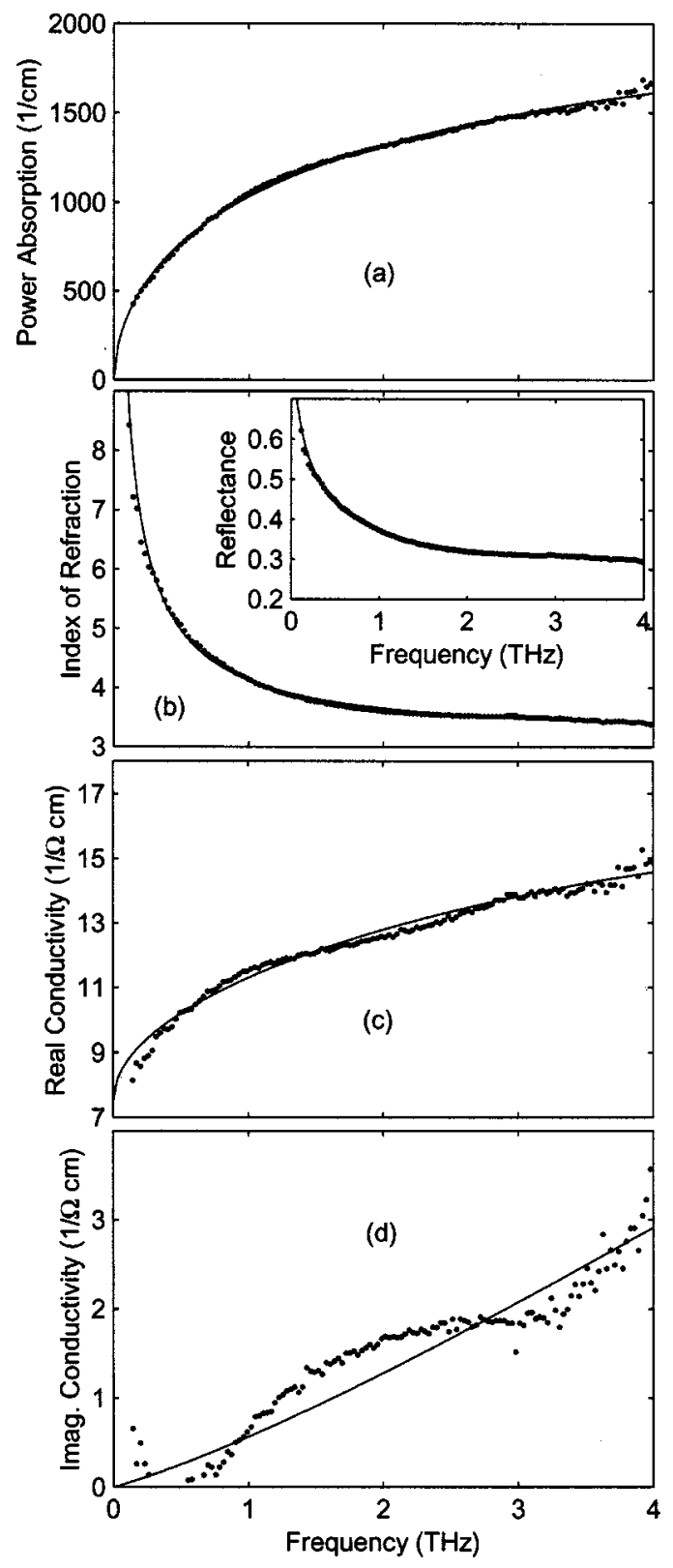

FIG. 2. Comparison of measurements (dots) with theory (solid line). (a) Power absorption coefficient $\alpha$. (b) Index of refraction $n_{r}$; inset, singlesurface reflectance $R$. (c) Real part of the conductivity $\sigma_{r}$. (d) Imaginary part of the conductivity $\sigma_{i}$.

plex amplitude transmission. The complex spectrum $E_{\text {ref }}(\omega)$ of the reference THz pulse [Fig. 1(a)] is related to the complex spectrum $E_{o}(\omega)$ of output THz pulse [Fig. 1(b)] by the equation $E_{o}(\omega) / E_{\mathrm{ref}}(\omega)=t(\omega) \exp \left[-i \beta_{0}(\omega)\right]$, where the term $\beta_{0}=2 \pi L / \lambda_{0}$ accounts for the air space displaced by the sample. Using the Fabry-Perot analysis of Eq. (1) and the measured ratio of $E_{o}(\omega) / E_{\text {ref }}(\omega)$, we can numerically remove the multiple reflection effects from our measured data and determine the power absorption $\alpha(\omega)$ and real part of the index of refraction $n_{r}(\omega)$ as shown in Figs. 2(a) and 2(b). The measured power absorption shows a smooth frequency dependence with the maximum measured value of $1600 / \mathrm{cm}$ at the highest frequency of our system. The measured index of refraction $n_{r}$ decreases rapidly in the low frequency range and then the slow monotonic decrease almost saturates at about $n_{r}=3.5$ in the high frequency range. The inset figure shows the power reflectance $R=\mid(1$ to AlP license or copyright, see http://ojps.aip.org/aplo/aplcr.jsp 
$-n) /\left.(1+n)\right|^{2}$ obtained from our measured data and theory, where $n=n_{r}+i n_{i}$. This reflectance is due to the first surface only and describes the thick sample case; it is presented to connect with the technique of reflective Fourier transform spectroscopy. ${ }^{1}$

Similar to semiconductors the conducting polymer's frequency-dependent dielectric response $\varepsilon(\omega)$ is assumed to be described by the general relationship,

$$
\varepsilon(\omega)=\varepsilon_{\text {Poly }}(\omega)+i \sigma(\omega) /\left(\omega \varepsilon_{0}\right)=\left[n_{r}(\omega)+i n_{i}(\omega)\right]^{2},
$$

where $\varepsilon_{\text {poly }}(\omega)$ is the dielectric constant of the undoped polymer, $\sigma(\omega)$ is the complex conductivity, and $\varepsilon_{0}$ is the free-space permittivity. Given the measured index data $n_{r}(\omega)$ and the absorption data $\alpha(\omega)$ which determines $n_{i}(\omega)$, and assuming that $\varepsilon_{\text {poly }} \approx(3.46)^{2}$, the square of the high frequency limit of the measured $n_{r}(\omega)$, then the real part $\sigma_{r}(\omega)$ and the imaginary part $\sigma_{i}(\omega)$ of the conductivity can be obtained from Eq. (2) as shown in Figs. 2(c) and 2(d). Clearly, our low conducting polymer sample, for which the real part of the conductivity $\sigma_{r}$ increases with increasing frequency, does not follow the simple Drude model behavior of $\sigma_{r}$, which has a Lorentzian line shape centered at zero frequency and with a half-width at half maximum (HWHM) at frequency $\Gamma /(2 \pi){ }^{8-10}$ As described below our measurements are consistent with the localization-modified Drude model.

The slight oscillation of the measured $\sigma_{r}$ with respect to the theoretical curve is due to an imperfect cancellation of the Fabry-Perot oscillations as described in Eq. (1) with the best fitting $n=n_{r}+i n_{i}$ obtained from Figs. 2(a) and 2(b). This could be due to thickness variations over the sample, improper centering of the sample aperture on the $\mathrm{THz}$ beam axis, or a slight phase variation across the incoming $\mathrm{THz}$ beam. This oscillation is more pronounced for the $\sigma_{i}$ measurement as shown in Fig. 2(d). However, for both cases the baseline of the oscillation is in good agreement with the theoretical curves.

For this more complex case, the complex conductivity $\sigma(\omega)$ was quantitatively analyzed in terms of the localization-modified Drude model, ${ }^{2,12}$

$$
\begin{aligned}
\sigma_{\mathrm{LD}}(\omega)= & \sigma_{\text {Drude }}(\omega)\left\{1-\left[C /\left(k_{F} \nu_{F}\right)^{2}\right] / \tau^{2}\right. \\
& \left.+(3 \omega)^{1 / 2}\left[C /\left(k_{F} \nu_{F}\right)^{2}\right] / \tau^{3 / 2}\right\},
\end{aligned}
$$

which involves the simple, frequency-dependent real function in the brackets multiplying the complex conductivity of the simple Drude model $\sigma_{\text {Drude }}(\omega)=i \varepsilon_{0} \omega_{p}^{2} /(\omega+i \Gamma)$. Using Eqs. (3) and (1), we obtained the localized power absorption $\alpha_{\mathrm{LD}}$ and real part of the index of refraction $n_{\mathrm{LD}}$, shown as the solid lines in Fig. 2, which are in excellent agreement with the measured data. The theoretical fit determined the three parameters: $\omega_{p} /(2 \pi)=25 \mathrm{THz}, \quad \Gamma /(2 \pi)=20 \mathrm{THz}$, corresponding to the carrier collision time of $\tau=8 \mathrm{fs}$, and $\left[C /\left(k_{F} \nu_{F}\right)^{2}\right]=0.36 \times 10^{-28} \mathrm{~s}^{2}$. For this parameter in brackets the individual terms are not separately determined. In conducting polymers, ${ }^{2} \mathrm{C}$ is expected to be of the order of unity;
$k_{F}$ is the Fermi wave vector, approximately $10^{9} \mathrm{rad} / \mathrm{m}$; and $\nu_{F}$ is the Fermi velocity, approximately $10^{5} \mathrm{~m} / \mathrm{s}$. The fit of the localization-modified Drude model of Eq. (3) to the data was basically by trial and error, initially guided by the asymptotic values of the experimental data and approximate two parameter fits to $\sigma_{\text {Drude }}(\omega)$. The very short time between collisions $\tau=8$ fs is approximately $1 / 15$ of that for the doped semiconductors $\mathrm{Si}$ and $\mathrm{GaAs}$ with similar conductivity and illustrates the disorder of the polymer. From Eq. (3) the dc conductivity can be obtained to be $\sigma_{\mathrm{LD}}(0)=7.5 /(\Omega \mathrm{cm})$, in reasonable agreement with the extrapolated dc conductivity from the THz-TDS measurement.

For the simple Drude model, the HWHM of the calculated real conductivity is equal to the collision frequency $\Gamma /(2 \pi)$. In both the localization-modified Drude model and the simple Drude model, the collision frequencies are located at the intersection of the real $\sigma_{r}$ and imaginary $\sigma_{i}$ parts of the conductivity, thereby providing a direct measure of the mobility $\mu=e /\left(m^{*} \Gamma\right)$ where $e$ is the electron charge and $m^{*}$ is the effective mass. As used in Drude theory $\omega_{p}^{2}$ $=N e^{2} /\left(\varepsilon_{0} m^{*}\right)$, where $N$ is the carrier density. The effective mass of electron is assumed to be $m^{*}=1.7 m_{e}$, the same as for conducting polypyrrole ${ }^{1}$ where $m_{e}$ is the free-electron mass. We can now calculate the very low mobility $\mu$ $=8.2 \mathrm{~cm}^{2} / \mathrm{V} \mathrm{s}$ and the carrier density $N=1.3 \times 10^{19} / \mathrm{cm}^{3}$. This mobility is significantly lower than $\mu=13 \mathrm{~cm}^{2} / \mathrm{V} \mathrm{s}$ previously obtained for the 30 times more conductive 215/ $(\Omega \mathrm{cm})$ polypyrrole film. ${ }^{3}$

In summary, the THz-TDS characterized $7.5 /(\Omega \mathrm{cm})$ sample of conducting poly-3-methylthiophene polymer film is well fit to the localized-modified Drude theory. This result is in contrast to a previous THz-TDS characterization of a much more conductive $215 /(\Omega \mathrm{cm})$ polypyrrole film, where the complex conductivity was in excellent agreement with Drude theory. Clearly, these experimental results await theoretical interpretation for these two different and high-interest conducting polymers.

The Oklahoma State part of this work was partially supported by the National Science Foundation and the Army Research Office.

\footnotetext{
${ }^{1}$ K. Lee, R. Menon, C. O. Yoon, and A. J. Heeger, Phys. Rev. B 52, 4779 (1995).

${ }^{2}$ K. Lee, A. J. Heeger, and Y. Cao, Phys. Rev. B 48, 14884 (1993).

${ }^{3}$ T.-I. Jeon, D. Grischkowsky, A. K. Mukherjee, and R. Menon, Appl. Phys. Lett. 77, 2452 (2000).

${ }^{4}$ S. Masubuchi, T. Fukuhara, and S. Kazama, Synth. Met. 84, 601 (1997).

${ }^{5}$ D. Grischkowsky, S. Keiding, M. van Exter, and Ch. Fattinger, J. Opt. Soc. Am. B 7, 2006 (1990).

${ }^{6}$ M. van Exter and D. Grischkowsky, Appl. Phys. Lett. 56, 1694 (1990); Phys. Rev. B 41, 12140 (1990).

${ }^{7}$ N. Katzenellenbogen and D. Grischkowsky, Appl. Phys. Lett. 61, 840 (1992).

${ }^{8}$ T.-I. Jeon and D. Grischkowsky, Phys. Rev. Lett. 78, 1106 (1997).

${ }^{9}$ T.-I. Jeon and D. Grischkowsky, Appl. Phys. Lett. 72, 2259 (1998).

${ }^{10}$ T.-I. Jeon and D. Grischkowsky, Appl. Phys. Lett. 72, 3032 (1998).

${ }^{11}$ M. Born and E. Wolf, Principle of Optics (Pergamon, Oxford, 1987).

${ }^{12}$ N. F. Mott and M. Kaveh, Adv. Phys. 34, 329 (1985).
} 